\title{
Beyond belief bias: Reasoning from conceptual structures by mental models manipulation
}

\author{
CARLOS SANTAMARÍA \\ Universidad de La Laguna, Tenerife, Spain \\ JUAN A. GARCÍA-MADRUGA \\ UNED, Madrid, Spain \\ and \\ MARIO CARRETERO \\ Universidad Autónoma de Madrid, Spain
}

\begin{abstract}
Mental models constitute an alternative to the rule-based systems in the explanation of human reasoning (Johnson-Laird, 1983). In this paper, we claim that the concept of believability generally used to categorize content and context effects is of little use within a semantic theory. Thus, we propose the use of categories that are directly extracted from subjective relations among concepts within the reasoning problem. We demonstrate that manipulations based on this kind of categorization produce predictable patterns of responses in reasoning problems. We present two experiments to test our predictions, using conditional and syllogistic reasoning problems, and in both cases, we demonstrate the influence of conceptual knowledge not only in natural contexts, but also in experimentally created artificial contexts.
\end{abstract}

Johnson-Laird's (1983) mental model theory proposes a semantic, non-rule-based approach to human reasoning. This theory is the only complete theory of deduction without explicit formal rules of inference. According to the mental model theory, human deduction depends on the construction and manipulation of analogical models in the mind. Model building and manipulation are processes that people carry out on line. Thus, models are not retrieved from long-term knowledge as rules or schemas are. The whole process of reasoning includes three stages (Johnson-Laird \& Byrne, 1991; see also Johnson-Laird \& Bara, 1984, for a previous version on syllogistic reasoning):

1. Comprehension: Reasoners build a mental model or a set of models from the state of affairs described in the phrasing of a problem.

2. Description: Includes the formulation of an informative but also parsimonious conclusion describing the existing model(s), and includes some information that is not explicit in the premises.

3. Validation: Subjects search for alternative models of the state of affairs, in which putative conclusions which have been already produced are false.

When alternative models which falsify the tentative conclusion are found in the third step, the system goes back to

Preparation of this article was partially supported by Grant $92 / 141$ from Gobierno de Canarias, Consejería de Educacion to C.S. and by Grant PB91-0028-C03-03 from DGCYT to M.C. We thank Phil Johnson-Laird for his helpful comments on an earlier version of this paper. Correspondence concerning this article should be addressed to C. Santamaria, Dpto. de Psicología Cognitiva, Universidad de La Laguna, Campus de Guajara, 38071 Tenerife, Spain (e-mail: csantamaria@ull.es). the second step to search for another tentative conclusion, and so on. Validation by searching for alternative models makes it possible to explain how a nonlogical system would be able to produce logically valid conclusions. If a persistent reasoner goes through all the steps, performing an exhaustive search for counter-examples, she/he would always give the correct conclusion. Only this exhaustive procedure would assure the production of a valid conclusion. When subjects have a complete representation of the elements in the problem and there is no simple conclusion that is true in all the models, the conclusion should be that Nothing follows. Reasoning capacity limitations are explained within this theory as a consequence of the limitations in the human processing capacity. For example, the limited capacity of working memory (see Baddeley, 1986) would restrict the number of possible models considered.

According to Johnson-Laird and Byrne (1991), mental models of the propositional connectives describe situations represented in the premises, but not necessarily in a completely explicit way: "They leave as much information as possible implicit in their models rather than spelling it out explicitly" (p. 43). For example, the initial models for a conditional, such as if $p$ then $q$, may be symbolized as (for simplicity, throughout this paper we will use the smallest number of elements representing the situation):

$$
\begin{aligned}
& p^{q} \\
& \ldots
\end{aligned}
$$

The first line represents a situation in which both $p$ and $q$ are true. For example, for a conditional such as If there is a prince, then there is a queen, the initial representation 
considers explicitly the situation in which there are both a prince and a queen, but it also considers elliptically that there could be other possibilities. The three dots in the second model represent the ellipsis. They indicate the model as a placeholder with no explicit content. In the initial model, $p$ and $q$ are not exhaustively represented. Therefore, both elements could occur anywhere else in the implicit models with affirmative or negative status (a prince could appear with or without a queen, and vice versa). Exhaustive representations are considered when a single-conditional or biconditional interpretation is carried out (JohnsonLaird \& Byrne, 1991):

$\begin{array}{cc}\text { Single Conditional } & \text { Biconditional } \\ {[p] \quad q} & {[p] \quad[q]} \\ \ldots & \ldots\end{array}$

-where square brackets stand for exhaustive representations. The exhaustively represented terms cannot be fleshed out with terms other than those present in the explicit model (e.g., since $q$ was not exhausted in the singleconditional initial model, it could occur in the explicit models with $p$ and not- $p$ ). Full fleshing out of models would only be done when necessary (when demanded by the task), and would be different for a material implication (singleconditional: if $p$ then $q$, but not necessarily if $q$ then $p$ ) and for a material equivalence (biconditional: if $p$ then $q$, and if $q$ then $p$ ). The items that were exhaustively represented in the initial model cannot appear elsewhere when the models are fleshed out. Therefore, the explicit representations would be as follows:

\begin{tabular}{ccrr} 
Single Conditional & \multicolumn{2}{c}{ Biconditional } \\
$p$ & $q$ & $p$ & $q$ \\
$\neg p$ & $q$ & $\neg p$ & $\neg q$ \\
$\neg p$ & $\neg q$ & &
\end{tabular}

- where the " $\neg$ " symbol represents negation. Exhaustive representation is necessary to ensure a valid conclusion.

The theory gives appropriate explanation for all the main psychological phenomena in conditional reasoning. For example, modus ponens inference (if $p$ then $q$; and $p$, therefore $q$ ) would be more frequently produced by reasoners than would modus tollens (if $p$ then $q$; and not- $q$, therefore not- $p$ ), because the former may be drawn directly from initial models and the latter depends on the fleshing out of models. Moreover, modus tollens requires two explicit models in the biconditional and three in the single conditional, and consequently, it is more frequently produced in biconditional (Johnson-Laird, Byrne, \& Schaeken, 1992). Facilitation by the content of the problem is expected to occur whenever a content induces the fleshing out of explicit models of negative instances.

Although this theory has been tested on a wide range of deductive tasks, the syllogism is probably the main task in which mental models theory has been applied. Syllogisms are expressions in the following form: all As are Bs and all $B s$ are $C s$; therefore, all As are $C s$-where the first two statements are called premises and the third is the conclusion. Each of the statements (premises and conclusion) contains a single quantifier (all, none, some, or some ... not), and the conclusion should necessarily follow from the premises.

Johnson-Laird and his colleagues (Johnson-Laird \& Bara, 1984; Johnson-Laird \& Steedman, 1978) have been interested in the way in which people produce conclusions from premises. In recent versions of the theory, the answer includes the concept of mental models (Johnson-Laird, 1983; Johnson-Laird \& Bara, 1984; Johnson-Laird \& Byrne, 1991). The first premise of a syllogism such as all $A s$ are $B s$ and all $B s$ are $C s$ produces an initial representation such as the following:

$$
\text { [a] } b
$$

-where each line represents an individual. Because quantified expressions make reference to sets of individuals, a line cannot represent a whole model as in propositional reasoning. Thus, each syllogistic premise calls for one model containing two sets of elements (Johnson-Laird \& Byrne, 1991). The remaining conventions are the same as in the conditionals. The explicit model of the same premise would take this form:

$\begin{array}{rr}a & b \\ \neg a & b \\ \neg a & \neg b\end{array}$

To represent both premises, the initial model of the second ( $A l l B s$ are $C S$ ) is added to the initial interpretation of the first premise:

$$
\left[\begin{array}{ll}
{[a]} & b
\end{array}\right] \quad c
$$

So, the fully fleshed-out representation is

$\begin{array}{rrr}a & b & c \\ \neg a & b & c \\ \neg a & \neg b & c \\ \neg a & \neg b & \neg c\end{array}$

This is a one-model problem, because there are no counterexamples to falsify the initial model. In other syllogisms, there may be two or three models. For this theory, the number of models is the main factor of difficulty in syllogistic reasoning. In fact, problems generating two or three mental models (those whose phrasing could describe more than a single situation) are more difficult than single-model problems (Johnson-Laird \& Bara, 1984).

Within this theory, as in most reasoning theories, thematic-content effects are explained mainly as a consequence of the believability of premises and conclusions (Oakhill, Garnham, \& Johnson-Laird, 1990; Oakhill \& Johnson-Laird, 1985; Oakhill, Johnson-Laird, \& Garnham, 1989). Beliefs might thus affect reasoning in three 
ways (see Oakhill et al., 1990): (1) by distorting the interpretation of the premises; (2) by biasing the process of deduction; and (3) by filtering the acceptation of unbelievable conclusions obtained during the process. Thus, if the putative conclusion is unbelievable, the subjects will search for alternative conclusions more frequently. However, if the initial tentative conclusion is believable, a search for falsifying models will be less likely to occur. According to this point of view, we might predict that content effects would be stronger in multiple-model problems. In other words, believability of a tentative conclusion may stop the search process, and the reasoner will therefore produce a (perhaps incorrect) conclusion. In contrast, the unbelievability of putative conclusions may encourage the search. However, Oakhill et al. (1989) failed to find any effect of beliefs on multiple-model-determinate syllogisms, whereas they found a reliable effect of beliefs on one-model problems. These results, which oppose the mental model theory predictions, forced the authors to provide an ad hoc explanation: "The determinate multiple-model problems are so difficult that subjects may be unsure that even a plausible conclusion follows and unsure whether they have checked out all the alternative models" (p. 136). Barston (1986) was also unable to find the desired effects of beliefs on multiple-model-determinate syllogisms in a construction task. Newstead, Pollard, Evans, and Allen (1992) found significant effects of beliefs on one-model problems, but not in multiple-model-determinate syllogisms. However, in all the similar experiments, the results have not been consistent. For example, Evans, Barston, and Pollard (1983) found belief effects in multiple-model problems in an evaluation task, and Santamaría (1989) found differences between single-model and multiple-model problems in belief effects (favorable for multiple-model problems). But these effects were only found in "positive" problems (i.e., problems with a believable and correct conclusion), whereas negative content effects were similar in single- and multiple-model problems.

A postreasoning conclusion filtering mechanism was introduced in the model theory of reasoning to account for these results: "subjects who are confronted with a valid but unbelievable conclusion, and can find no way of representing the premises that refutes it, altered the conclusion in some way" (Oakhill \& Garnham, 1993). This explanation has been criticized by Newstead and Evans (1993) because of its arbitrariness. Moreover, it limits the refutability of the theory. If the theory predicts a response to a given problem, and if an external mechanism can twist the response in some way after the reasoning process, then the predictions of the theory are useless in testing its validity.

It may be possible to argue that both the inconsistency of the results in multiple-model-determinate problems and the effect of beliefs in one-model syllogisms constitute arguments against the mental model theory. However, from our point of view, they are only methodological consequences of the use of an inappropriate categorization of beliefs. The contents of syllogisms have been categorized in most experiments as believable, unbelievable, and, sometimes, neutral. In this paper, we present a different categorization based on the subjective relations among the concepts included in the problems. Consequently, we will try to demonstrate how the hypotheses obtained with this kind of categorization are more specific than those derived only from believability. The experiments designed from this categorization clearly support the mental model theory. In our opinion, the possible subjective relations among concepts are more than just believable, unbelievable, and neutral. The subjects can make a number of different interpretations of the semantic relationships within a set of concepts. These interpretations depend on the longterm knowledge and the context, and have an influence on the construction and manipulation of mental models. The initial model for premises and, consequently, the fleshingout process are affected by the reasoner's interpretation of the relationship among the concepts. We maintain that five categories of interpretation are especially useful in the study of reasoning:

1. Identity. Given two categories $A$ and $B$, we can interpret them as being identical if the subjects suppose that all the members of set $A$ are also members of set $B$ and vice versa. For example, this may be the typical interpretation of the relationship between the concepts jersey and jumper. This kind of representation is equivalent to a possible interpretation of quantified expressions such as All As are $B s$ (traditionally labelled $A$ ). When people grasp that the two terms are coextensive (e.g., in the statement All jerseys are jumpers), they would need both terms to be exhausted:

$$
\text { [jersey] [ jumper] }
$$

This procedure is similar to that explained for the biconditionals. In fact, identity is also consistent with the material equivalence in conditional reasoning. The previous model could be fleshed out as

$$
\begin{array}{cc}
\text { jersey } & \text { jumper } \\
-j e r s e y & -j \text { jumper }
\end{array}
$$

-where the case of a jersey that is not a jumper is not considered.

2. Disjunction. This refers to the case in which the interpretation that people carry out would suppose that no member of set $A$ was also a member of set $B$ and vice versa (e.g., chair and table). The only syllogistic relation equivalent to this type of context is an expression like No As are Bs $(\mathrm{E})$. According to the mental model theory, this produces an initial representation such as

$$
\text { [a] }
$$

and with a disjunction context such as No chair is a table, the model is fleshed out as 


$\begin{array}{cr}\text { chair } & \neg \text { table } \\ \neg \text { chair } & \text { table } \\ \text {-chair } & \text { table }\end{array}$

3. Intersection. With this case, people suppose that some but not all the members of set $A$ are also members of set $B$ and vice versa (e.g., waiter and tall people). Two types of quantified expressions could be equivalent to this kind of context: Some As are Bs (I) and Some As are not Bs (O). The first type of expression is initially represented as

$$
a
$$$$
b
$$

$$
\text { ... }
$$

while the representation of the second is

$$
a
$$$$
a
$$

Regardless of the order of the elements in the model, both expressions with an intersection context (e.g., Some waiters are tall people and Some waiters are not tall people) could be fleshed out to yield an explicit representation like this:

$\begin{array}{rr}\text { waiter } & \text { tall person } \\ \text { waiter } & \neg \text { tall person } \\ \neg \text { waiter } & \text { tall person } \\ \neg \text { waiter } & \neg \text { tall person }\end{array}$

4. Direct inclusion. This interpretation shows that all the members of set $A$ are also members of set $B$, but that only some of the members of set $B$ are members of set $A$. Obviously, this interpretation is not symmetrical (e.g., French and European). We use the term direct inclusion to refer to the cases in which the first concept $(A)$ is included in the second $(B)$, and we use the term inverse inclusion for the cases in which $B$ is included in $A$. Direct inclusion is equivalent to the logical relation affirmative universal (e.g., All the French are European), which in this case could be initially represented as

$$
\text { [French }] \quad \text { European }
$$

and the representation could be fleshed out as

$$
\begin{array}{rr}
\text { French } & \text { European } \\
\neg \text { French } & \text { European } \\
\neg \text { French } & \neg \text { European }
\end{array}
$$

This relation is also consistent with the material implication in conditional reasoning.

5. Inverse inclusion. Its representation is equivalent to both I and O relations (e.g., Some Europeans are French and Some Europeans are not French). In these cases, once subjects grasp that the second term is included in the first term, the model-like representation could be

$$
\text { European [French }]
$$

- where only the second term is exhausted. Consequently, the representation could be fleshed out as

$\begin{array}{rr}\text { European } & \text { French } \\ \text { European } & \neg \text { French } \\ \neg \text { European } & \neg \text { French }\end{array}$

Each of these five categories represents extreme cases that could be found when one is using well-defined concepts (intermediate states may exist). We call the exact matching between the interpretation of the relationship and a given logical relation functional equivalence. We maintain that this phenomenon is responsible for the different proportion of logically correct and incorrect responses found in reasoning experiments when content and context manipulations are used.

Five categories of interpretation (identity, disjunction, intersection, direct-inclusion, and inverse-inclusion) have been presented. We call them typical concept relationships representations (TCRRs). In our presentation of the five TCRRs we have also shown the quantified expressions that could be believable in each case. Believability arises from the functional equivalence between TCRR and the structure of the problem. Table 1 summarizes this correspondence. For example, two concepts maintaining a disjunctive

\begin{tabular}{|c|c|c|c|c|c|}
\hline & Identity & Disjunction & Intersection & $\begin{array}{c}\text { Direct } \\
\text { Inclusion }\end{array}$ & $\begin{array}{l}\text { Inverse } \\
\text { Inclusion }\end{array}$ \\
\hline Single conditional & & & & $x$ & \\
\hline Biconditional & $x$ & & & & \\
\hline All $A$ are $B$ & $x$ & & & $\times$ & \\
\hline No $A$ are $B$ & & $x$ & & & \\
\hline Some $\mathrm{A}$ are $\mathrm{B}$ & & & $x$ & & $x$ \\
\hline Some $A$ are not B & & & $x$ & & $\times$ \\
\hline
\end{tabular}
TCRR will only be believable in universal negative expressions (e.g., No dogs are cats); therefore, a universal affirmative with the same concepts will be unbelievable (i.e., All dogs are cats). The concept of believability is a less specific term than TCRR. For example, identity and direct inclusion are both believable in universal affirmatives, but different interpretations are predicted from

Table 1

The Relationship Between Believability and TCRRs in Conditionals and Quantified Relations

Note-The Xs indicate functional equivalences. 
TCRR. Therefore, the empirical relations between concepts should replace believability as an independent variable in reasoning experiments.

Two features of TCRR should be noted. The first feature is implied in the subjective nature of TCRR. All the categorizations are based on subjects' interpretations of the statements, so in spite of the logical meaning of the existential quantifiers (i.e., at least one, but possibly all), identity, direct inclusion, and disjunction are not considered when one is dealing with existential propositions (those including the quantifiers: some or some ...not). Consequently, I (existential affirmative) and $\mathrm{O}$ (existential negative) statements share the same conceptual interpretations and are equivalents from the point of view of TCRR (however, inclusion could be a more frequent interpretation for $\mathrm{O}$ statements, because of its asymmetry), even though other factors may differ (e.g., initial models are different). Second, different TCRRs can be functionally equivalent to the same logical structure for the initial representation. For example, as noted, in universal affirmatives both identities and inclusions may be believable. However, their fleshed-out mental models may differ. As shown in the description of identity and direct inclusion, the fleshed-out model of the A-identity believable statement all jerseys are jumpers is less likely to include the case of a jumper that is not a jersey than is the A-inclusion also believable statement all French people are European to include the case of a European who is not French.

These kinds of differences in the interpretation would produce different conclusions. From a semantic mental model theory like that of Johnson-Laird these effects are predictable and do not need to be explained as effects of misinterpretations but as combinations between TCRR and problem structure. Thus, content effects are predicted even in single-model problems whenever they include differences in TCRR. Indeed, since all the single-model syllogisms include at least one A premise yielding two different TCRRs, it is easier to produce content-based differences in problems of this nature, and this could be the reason why most authors find such effects. The most difficult conclusion to induce through the manipulation of TCRR is the negative existential, because, as noted, it shares TCRR with the affirmative existential, and a mental model consistent with an $\mathrm{O}$ conclusion should also be consistent with an I conclusion when TCRR is optimized (not necessarily when some elements in the model are more salient than others, as may occur with poorly defined concepts). Multiplemodel-determinate syllogisms always have an O conclusion. Therefore, any manipulation in an attempt to produce the correct conclusion in such problems could also elicit the incorrect I conclusion. This difficulty could explain the inconsistent results of the experiments that manipulate believability in problems of this kind.

The idea that, depending on real-world knowledge, there may be different interpretations of a given statement is well known within the field of conditional reasoning (for a review, see Evans, Newstead, \& Byrne, 1993). Although the majority of the studies on content and context effects in this field use the Wason (1966) selection task, and have mostly been concerned with the manipulation of believability (see Evans, 1989), other studies have directly manipulated the meaning of the conditional as a function of the context. The reason probably is that formal logic itself attributes two different meanings for conditional statements (i.e., implication and equivalence). Consequently, even mental-logic theories consider the possibility of different valid interpretations of conditionals, depending on the context. For example, Legrenzi (1970) found that in a binary situation, interpretation of the conditionals appears to include its converse. Rips and Marcus (1977; cf. Marcus \& Rips, 1979) replicated the experiment with both the selection task and a truth-table task, and found analogous results. Marcovits (1984; also 1985, 1988) found that the ability of the subjects to imagine alternative situations could block the fallacies (affirming the consequent and denying the antecedent). Particularly, if the subjects consider more than one possibility, they mostly infer according to material implication, whereas if they consider a single possibility, their responses tend to map a material-equivalence pattern. Byrne (1989) demonstrated that this effect is not specific for fallacies. By including additional premises, she blocked modus ponens and modus tollens inferences. This finding, which contradicted the distinction between necessary and invited inferences (Braine, 1978; Braine \& Rumain, 1983; Osherson, 1975), supports the idea that the way in which information from different sources is integrated in mental models depends on general knowledge.

Our purpose is to demonstrate that both conditional and syllogistic performance is affected by TCRR in the same way. Most research on conditional reasoning has been concentrated on two different interpretations of if ... then ... statements: material implication and material equivalence. From our point of view, these interpretations correspond, respectively, to two of the possible TCRRs, inclusion and identity, so the interpretation is the same as it is in universal affirmative quantified expressions (e.g., if he is a Frenchman, then he is a European is functionally equivalent to a single conditional, and if it is a jumper, then it is a jersey would be interpreted as a biconditional).

We tested our predictions in two experiments in which we used artificially induced conceptual relationships in conditional reasoning (Experiment 1) and natural concepts with different TCRRs in syllogistic reasoning (Experiment 2).

\section{EXPERIMENT 1}

To test functional equivalence predictions in conditional reasoning, and from artificial contexts, we gave subjects two contexts that were, respectively, functionally equivalent to the single conditional and the biconditional. An ambiguous control context was also used. For all contexts, the rationale stated the existence of two bags containing white and black balls. In the single-conditionalequivalent condition (inclusion condition), subjects were told, "There are white and black balls in Bag A and only black or white balls in Bag B." When the conditional rule If the ball is white, it is in Bag $A$ is added, the mental model created would be similar to that of inclusion, which, as 
previously mentioned, is functionally equivalent to the single conditional. This is because it is clear that there are both white and nonwhite (black) balls in Bag A. In the biconditional equivalent version (identity condition), subjects were told, "There are only white or black balls in each bag," but the same conditional rule was used. The mental model would now be equivalent to the biconditional. According to the TCRR of inclusion and identity (see above), the fleshed out representation of each condition would be

$$
\text { Inclusion }
$$$$
\text { Identity }
$$

$\begin{array}{llll}\text { white ball } & \text { Bag } A & \text { white ball } & \text { Bag } A \\ \text { black ball } & \text { Bag } A & \text { black ball } & \text { Bag } B \\ \text { black ball } & \text { Bag } B & & \end{array}$

-which is functionally equivalent to the fleshed out models for, respectively, the single conditional and the biconditional. Therefore we predict that each context will produce the same type of responses that would be drawn by a system with the corresponding logical mechanism; modus ponens (MP) and modus tollens (MT) in inclusion, and MP, MT, denying the antecedent (DA) and affirming the consequent (AC) in identity. A wide pattern of responses is expected for the control context - but with a dominance of only-MP interpretations, because this seems to be the more parsimonious conclusion when there is not much information.

\section{Method}

Subjects and Design. A total of 120 psychology and education students at Universidad de Málaga ( 93 females and 27 males) received one of three tasks. The three tasks were distributed at random, and 40 subjects were assigned to each condition. The mean age of subjects was 22.48 years, and the standard deviation was 4.89 .

Materials and Procedure. The three tasks were distributed at random among entire classes of students, so that general instructions about the nature of the task were shared by all the experimental conditions. Afterward, the subjects received the task form. At the top of the page, they found a description of the distribution of a set of balls in two given bags. Depending on the experimental condition, this rationale stated (all the instructions in this paper are translated from Spanish):

Inclusion: Imagine we have two bags containing black and white balls. In Bag $A$, there are white and black balls. In Bag B, there are only balls of one color: either black or white.

Identity: Imagine we have two bags containing black and white balls. One of them contains only white balls, and the other. only black balls.

Neutral: Imagine we have two bags containing black and white balls. We will call them Bag $A$ and Bag $B$.

All subsequent explanations were exactly the same for all the conditions. In order to test whether the different rationales produced different interpretations of the task context, four questions were asked, each having three options:

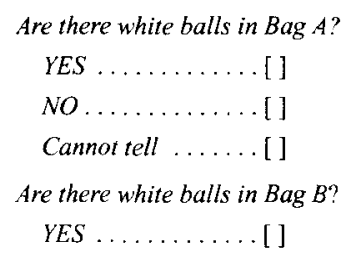

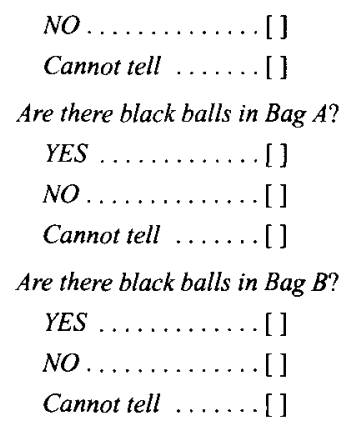

Following these questions, subjects found the statement "Mark an option for each question, which you think may be correctly drawn, taking into account the following rule." In the next line they read the rule: "If the ball is white, it is in Bag A" and the four cases traditionally named $p$, not- $p, q$, and not-q. Each of them followed three response options:

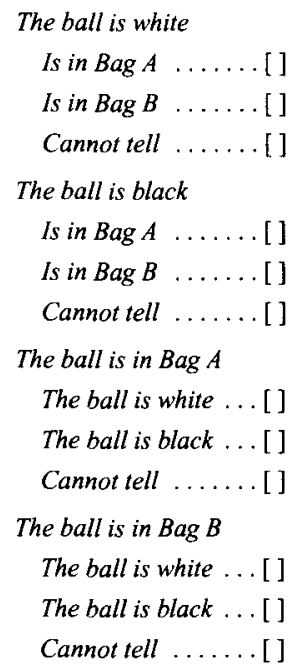

\section{Results}

Table 2 shows the response combinations in each experimental condition. The different effects induced by experimental conditions are clearly shown in this table. The analyses were performed on the probability of each experimental condition to produce each of the predicted patterns. We tested the hypothesis for the combination MP-MT, the implication pattern, the MP-MT-AC-DA (equivalence pattern), and only-MP, which we could refer

Table 2

Percentages of Response Combinations in Each Experimental Condition

\begin{tabular}{lccc}
\hline & \multicolumn{3}{c}{ Experimental Condition } \\
\cline { 2 - 4 } \multicolumn{1}{c}{ Combination } & Inclusion & Identity & Neutral \\
\hline MP & 17.5 & 0.0 & 35.0 \\
MP-MT & 27.5 & 0.0 & 7.5 \\
MP-AC & 17.5 & 0.0 & 20.0 \\
MP-AC-DA-MT & 15.0 & 97.5 & 27.5 \\
\hline Note-C &
\end{tabular}

ns with an overall frequency less than or equal to 2 were not included in the table. This affected $11.7 \%$ of the subjects; however, they were included in the calculus of percentages. MP, modus ponens; MT, modus tollens; AC, affirming the consequent; DA, denying the antecedent. 
to as the "conservative" response. For each of these patterns we created a binary variable, scoring 1 for the presence and 0 for the absence of the corresponding pattern. Although resulting data are dichotomous, we performed an analysis of variance on them (see Scheffé, 1960). With the MP-MT pattern, we found a significant difference between the conditions $[F(2,117)=8.80, p<.0005]$. Post hoc comparisons showed that the inclusion condition produced significantly higher scores than did the identity and the neutral conditions. In the MP-MT-AC-DA pattern, a significant difference was also found $[F(2,117)=$ $65.86, p<.0001]$. Post hoc comparisons revealed significantly higher scores in the identity condition than in the other two conditions. The only-MP pattern also registered significant differences $[F(2,117)=9.63, p<.0005]$. In this case, post hoc comparisons reached significance only for the contrast between neutral and identity conditions. As previous contrasts were performed on nonindependent variables (they all came from the same set of responses), we calculated the probability of making one or more Type I errors in the set of comparisons by applying the Bonferroni test. The probability of making this error was significantly low $(p<.001)$, so we can reject the hypothesis of a cumulated Type I error.

Table 3 presents another categorization of responses. In this table, we present the results for each of the conditional inference rules separately. It clearly shows that MP was produced by almost every subject in all experimental conditions. The other rules were consistently used in the identity condition, and less frequently in the other conditions. There also seems to be an interaction between the inclusion and the neutral conditions in MT and AC. This interaction is consistent with the significant differences mentioned above. The affirmative rules (MP and AC) were more frequent in both the inclusion and the neutral conditions than were the negative ones (MT and DA). No statistical test of significance was performed on the data presented in this table, because it is only a new categorization of data reported in Table 2.

Subjects' interpretation of the color of balls in each bag, based on the initial rationale, is reported in Table 4. As shown in the table, all subjects in the inclusion condition interpreted that there were black and white balls in Bag A and that the colors of the balls in Bag B were unknown. Most subjects in the identity condition reported that they could not tell anything about the color of balls in each bag,

Table 3

Percentages of Acceptance of Each Inferential Rule in Each Experimental Condition

\begin{tabular}{cccc}
\hline & \multicolumn{3}{c}{ Experimental Condition } \\
\cline { 2 - 4 } Combination & Inclusion & Identity & Neutral \\
\hline MP & 95.0 & 100.0 & 100.0 \\
MT & 50.0 & 100.0 & 42.5 \\
AC & 37.5 & 100.0 & 52.5 \\
DA & 22.5 & 97.5 & 35.0 \\
\hline
\end{tabular}

Note-MP, modus ponens; MT, modus tollens; AC, affirming the consequent; DA, denying the antecedent.
Table 4

Percentages of Combined Interpretations on the Distribution of Balls in Each Bag in Each Experimental Condition

\begin{tabular}{cccccc}
\hline \multicolumn{2}{c}{ Interpretation } & & \multicolumn{3}{c}{ Experimental Condition } \\
\cline { 5 - 6 } Bag A & Bag B & & Inclusion & Identity & Neutral \\
\hline b\&w & $?$ & & 100.0 & 0.0 & 2.5 \\
$?$ & $?$ & & 0.0 & 65.0 & 40.0 \\
w & b & 0.0 & 35.0 & 0.0 \\
b\&w & b\&w & 0.0 & 0.0 & 52.5 \\
\hline
\end{tabular}

Note-The first column represents the distribution of balls in Bag A. The second column does so for Bag B. In the table, "b" means black balls, and "w," white balls. A "?" shows when subjects stated that they could not tell the color of the balls in a bag. Interpretations with an overall percentage less than 2 were not reported. Only two responses (different from each other) were eliminated by this criterion (both in the neutral condition).

but some of them interpreted that there were only white balls in Bag A and only black balls in Bag B. In the neutral condition, the interpretation pattern was less clear. However, the majority of subjects identified either that there were white and black balls in both bags or that they could not tell. Only 1 subject in the neutral condition made the characteristic interpretation in the inclusion condition.

\section{Discussion}

Our predictions were satisfied in this experiment. Among most subjects, the identity and inclusion conditions led, respectively, to biconditional and single conditional. The neutral condition adopted an intermediate position in both cases. Hence, the MP-MT combination was less frequent in the neutral than in the inclusion condition, but more frequent than in the identity condition, whereas the MP-MTDA-AC combination worked in the opposite way. This confirms the prediction that when no specific clues are provided, the subjects' responses are less predictable. The most common response in the neutral condition was onlyMP. This direct inference matches all possible interpretations of conditional statements and hence is parsimonious when little is known about the circumstances to which the task is related, but the difference with respect to inclusion was not significant. This weaker effect was probably due to the predicted greater dispersion of responses in this condition. Another frequent interpretation in the neutral condition is the biconditional equivalent. It may be interpreted that when no other information is present, subjects tend to interpret abstract sets as disjointed. Therefore, in this case, the representation would be equivalent to that of the identity condition.

Another interesting result was that individual rules were more commonly accepted when they were affirmative (MP and $\mathrm{AC}$ ) than when they were negative (MT and DA). This result is consistent with the mental model theory. JohnsonLaird et al. (1992) explained the greater frequency of MP than of MT. In the case of AC and DA, the results varied across different studies (Evans, 1993). However, JohnsonLaird (1995) considers that this inconsistency is due to different ways of fleshing out the models. In our identity condition, which promoted biconditional interpretations, 
the proportion was similar for both inferences, whereas in the inclusion condition (single conditional), AC and DA were not found to be very frequent. Since $A C$ was more frequent than DA in our inclusion and our neutral conditions, the proposal of Evans on the existence of a bias in favor of negative conclusions was rejected by our data.

Results referring to previous interpretations of the situation of balls in each bag should be interpreted as exploratory. The interpretation is, in general, consistent with our goal. The only odd thing about these results is that $35 \%$ of the subjects in the identity condition reported that there were only white balls in Bag A and only black balls in Bag B. This reflects the reluctance of subjects to report that they cannot tell in all the cases, as should have been done in this condition. So this would suggest that subjects searched for other clues and read the conditional rule below on the same page. This task-design problem had no influence on the reasoning task, because both situations yield an interpretation equivalent to biconditional.

A context manipulation was performed in this experiment. The manipulation was based on TCRR, and reliable effects were found. These differences were not predicted by believability because the rule If the ball is white, it is in $\operatorname{Bag} A$ is believable in the three different domains. TCRR is the only categorization system for content and context predicting different responses for equally believable statements. From a logical point of view, it could be argued that the differences between the identity and inclusion conditions are solved with different rules: those of material equivalence and material implication, respectively. Although such an approach cannot easily explain the results in the neutral condition, it is not completely rejected by this experiment with conditional statements. In the next experiment we tested our predictions in syllogistic reasoning, where no alternative interpretations are predicted from formal rules of inference.

\section{EXPERIMENT 2}

In Experiment 1, we demonstrated how TCRR may be used to predict the kind of responses given in reasoning problems. In Experiment 2, we attempted to satisfy two main purposes: first, generalization of results to a different reasoning task (syllogistic reasoning), and second, the use of natural concepts and conceptual relations from the subjects' long-term memory, instead of artificial relations, as a means of testing our predictions about TCRR in a more natural context. Three thematic conditions and an abstract control condition were used, and the syllogism premises presented had the same mode for all the conditions:

\section{Some $A$ are $B$}

\section{No $X$ are $B$}

In thematic conditions, only the term in position $X$ changed across the conditions, so in these three conditions the problem was as follows:

Some students take examinations in September

No $\{X\}$ takes examinations in September
Again, TCRR categories were used to establish experimental conditions. These categories concerned the relationship between the extreme term in the first premise (student) and the extreme term in the second premise (the value of $X$ ). Since the first premise represents an inclusion, its fleshed-out model would be

$\begin{array}{rr}\text { student } & \text { september } \\ \text { student } & - \text { september } \\ \neg \text { student } & - \text { september }\end{array}$

We predicted that each of the empirical relations among terms in the syllogism would lead subjects to build different mental models and provide responses according to each of the different models. In this sense, disjunction ( $X=$ teacher $)$ would mainly produce the following fleshed-out model:

$\begin{array}{ccc}\text { student } & \text { september } & \neg \text { teacher } \\ \text { student } & \neg \text { september } & \neg \text { teacher } \\ \neg \text { student } & \neg \text { september } & \text { teacher } \\ \neg \text { student } & \neg \text { september } & \neg \text { teacher }\end{array}$

The most frequent conclusion would be that of the universal negative form $(\mathrm{E})$. The model most frequently built in the intersection condition ( $X=$ blond people) would be

$\begin{array}{rrr}\text { student } & \text { september } & \neg \text { blond } \\ \text { student } & \neg \text { september } & \text { blond } \\ \text { student } & \neg \text { september } & \neg \text { blond } \\ \neg \text { student } & \neg \text { september } & \text { blond } \\ \neg \text { student } & \neg \text { september } & \neg \text { blond }\end{array}$

This would produce particular responses, both affirmative and negative, and inclusion ( $X=$ bookworm) would mainly produce the following fleshed out model:

$\begin{array}{ccc}\text { student } & \text { september } & \neg \text { bookworm } \\ \text { student } & \neg \text { september } & \text { bookworm } \\ \text { student } & \neg \text { september } & \neg \text { bookworm } \\ \text { student } & \neg \text { september } & \neg \text { bookworm }\end{array}$

-where, since there are no cases of bookworms that are not students, the TCRR between the extreme terms is an inverse inclusion. (The word bookworm is used here as an approximate translation of the Spanish word empollón used in the experiment. This word is used among students to designate a student who studies a lot; it is similar to the British word swot. Consequently, all the empollón people are students, whereas some bookworms are not students. All the subsequent interpretations assume the Spanish meaning of the word.) Thus, particular and negative responses in the sense set-subset are expected-in other words, from the extreme term in the first premise to the extreme term in the second premise. Since abstract sets are interpreted as disjointed by default, the abstract condition would work in a similar way to disjunction and therefore would lead mainly to universal-negative responses. 


\section{Method}

Subjects. We tested 1391 st-year psychology students from the Universidad Autónoma de Madrid. One hundred eleven of them were female, and 28 were male. The mean age of the subjects was 19.32 years, and the standard deviation was 1.67 years.

Design. A between-subjects design was used. There were four experimental groups, one for the abstract problem and three for the thematic conditions (inclusion, disjunction, and intersection). A total of 36 subjects were randomly assigned to the abstract group; to the inclusion group, 34; to the disjunction group, 34; and to the intersection group, 35 .

Materials and Procedure. As in the first experiment, tasks were distributed at random among entire classes of students, who shared the same verbal instructions. These instructions included abstract examples using letters (A, B, and C). No example was from the same mode as that of the experimental problem. In the written instructions the subjects found the reminder that the middle term should not be included in the conclusion. Next, the four possible relations for the conclusion were reported (always using letters A, B, and $C$ ). These four examples were presented in random order for each subject. Finally, they located the syllogism. According to the manipulation of extreme term concepts, we obtained the following four problems:

$$
\begin{aligned}
& \text { Abstract: } \\
& \text { Some } Z \text { are } X \\
& \text { No J is } X \\
& \text { Inclusion: } \quad \begin{array}{r}
\text { Some students take examinations in September } \\
\text { No bookworm takes examinations in September }
\end{array} \\
& \text { Disjunction: Some students take examinations in September } \\
& \text { No teacher takes examinations in September }
\end{aligned}
$$

The subjects then had to write the conclusion. They were given no time limit.

\section{Results}

Table 5 presents the type of response occurrences for each experimental condition. In order to test our main hypothesis, we grouped the responses as predicted, or not. Moreover, in the abstract and disjunction conditions, the predicted responses were any using the structure $N o$... is ... In the intersection condition the predicted responses were those of the structure Some ... are ... or Some ... are not ... In the inclusion condition, since the corresponding TCRR is not symmetrical, there was only one type of predicted

Table 5

Percentages of Responses Corresponding to Each Relation for Each Experimental Condition

\begin{tabular}{lrccc}
\hline & \multicolumn{4}{c}{ Experimental Condition } \\
\cline { 2 - 5 } Response & Abstract & Intersection & Inclusion & Disjunction \\
\hline I (1st-2nd) & 8.3 & 28.6 & 17.6 & 5.9 \\
O(1st-2nd) & 27.8 & 37.1 & 64.7 & 26.5 \\
O(2nd-1st) & 0.0 & 0.0 & 0.0 & 8.8 \\
E (1st-2nd) & 41.7 & 17.1 & 14.7 & 35.3 \\
E (2nd-1 st) & 22.2 & 8.6 & 0.0 & 17.6 \\
MTI & 0.0 & 8.6 & 2.9 & 5.9 \\
\hline
\end{tabular}

Note-- $\mathrm{I}$, Some As are Bs; $\mathrm{O}$, Some As are not Bs; E, universal negative; MTI, middle term included in the conclusion; $1 \mathrm{st}-2$ nd, conclusions from extreme term in first premise to extreme term in second premise; 2nd-1st, opposite direction. response: Some ... are not ..., in the sense that goes from the extreme term in the first premise to the extreme term in the second premise. Since there were eight possible responses to the problem (the four relations multiplied by the two senses; when the middle term was included in the conclusion, the response was considered missing), the expected proportion of the predicted responses for the conditions with bidirectional hypothesis (abstract, intersection, and disjunction) was .25 , while for the condition with only one predicted response (inclusion) the expected proportion was .125. A chi-square test was carried out contrasting the expected proportion against the observed proportion in each condition. These tests reached significance in all the conditions: abstract $\left[\chi^{2}=29.04, p<.0001\right]$, intersection $\left[\chi^{2}=\right.$ $37.50, p<.0001]$, inclusion $\left[\chi^{2}=88.52, p<.0001\right]$, and disjunction $\left[\chi^{2}=16.67, p<.0001\right]$. An overall effect of the predicted responses was also found, when considering together all the experimental conditions [expected proportion $\left.=.219 ; \chi^{2}=142.47, p<.0001\right]$.

The majority of the conclusions produced were drawn in the following direction: first, the extreme term in the first premise; and then, the extreme term in the second premise. The percentage of responses was 83.1 for all of the conditions $\left[\chi^{2}=65.03, p<.0001\right]$. Each of the experimental conditions presented a significant effect when tested individually: abstract, $77.8 \%\left[\chi^{2}=11.11, p<.001\right]$; intersection, $90.6 \%\left[\chi^{2}=21.13, p<.0001\right]$; inclusion, $100 \%$ $\left[\chi^{2}=33.00 ; p<.0001\right]$; disjunction, $71.87 \%\left[\chi^{2}=6.12\right.$, $p<.05]$.

\section{Discussion}

On the conclusions drawn by subjects, we have demonstrated the influence of TCRR between the extreme terms of the syllogism. Again, we predicted conclusions in a reasoning problem using TCRR in combination with the mental models. Our predictions were clearly supported by conclusions in all the experimental conditions. The abstract condition showed the disjunction pattern of responses even more clearly than did the disjunction condition itself. This result may seem surprising, but empirical relations between concepts are not objective. Some people may think that some teachers are students (perhaps taking postgraduate courses). These subjects will produce particular responses to the problem. Abstract problems allow subjects to retrieve the relation that they consider useful for the particular problem.

As in Experiment 1, the analysis focused on the type of response that subjects draw from a given state of affairs. This is a methodological change with regard to measuring (as usual) the number of "correct" responses for each content manipulation condition. From this traditional point of view, the only information gleaned from this experiment would be that the inclusion is the condition in which the number of logically correct responses is more notable. From a psychological (nonlogical) point of view, this information is not very relevant, since the type of conclusion is more informative about cognitive processes than is the proportion of logically correct responses. 
Another advantage of the categorization in terms of TCRR has been made clear in this experiment. Some students are not blond is as believable as Some students are not bookworms, so the clear differences found are not explainable in terms of the believability of the conclusion.

\section{GENERAL DISCUSSION}

In this paper, we have presented two experiments that illustrate a new categorization of content and context effects in reasoning. We demonstrated that the hypotheses constructed from this point of view produce measurable effects that can be explained from within the theory of mental models. In the mental model theory, we must avoid the identification of difficulty with the proportion of logically incorrect responses. Indeed, there may be relatively easy cognitive tasks that lead to invalid conclusions. By arguing that people build different mental models, depending not only on the syntactic structure of the problem, but also on the concepts involved, the most significant variables were included in the reasoning process itself.

Other theories, such as pragmatic reasoning schemas (Cheng \& Holyoak, 1985), make use of specific reasoning rules for particular domains. Mental model theory is more ambitious. It tries to explain all the reasoning phenomena from the same set of mechanisms, both in abstract and concrete problems. One of the best features of the mental model theory is that its explanatory power appears in both reasoning and discourse comprehension. A mental model is supposed to be a representation of a given state of affairs. Glenberg, Meyer, and Linden $(1987$, p. 70$)$ pointed out that "a mental model is a representation of the situation described by a text, what the text is about, rather than a representation of the text ... itself" (see also Garnham, 1981, and Johnson-Laird, 1983). Also, a mental model for a reasoning problem comes from the situation described by the problem, not just from the structure of the problem. This situation may be artificially created by the experimenter (as in our Experiment 1), or based on the previous knowledge of the subject (Experiment 2). In this paper, we established the same types of interpretations for conditional statements and affirmative universal expressions. Formalrule theories consider both interpretations of conditionals. The identity interpretation of both the affirmative universals and the conditionals are supposed to include invited inferences (see Rumain, Connell, \& Braine, 1983), so they constitute misinterpretations. From a mental model theory viewpoint, the same kind of explanation is given for conditional and syllogistic problems. We have presented in this paper the nature of the correspondence of the different interpretations in both types of problems.

Content and context of reasoning problems have been operationalized to date in dichotomous categories: believable versus unbelievable (used especially in syllogistic reasoning: e.g., Evans et al., 1983; Oakhill et al., 1989), and familiar versus abstract (more common in conditional reasoning: e.g., Griggs \& Cox, 1982; Manktelow \& Evans, 1979). These dichotomies produce hypotheses with two values: biasing and debiasing (see Evans, 1989). These concepts are not independent of the logical validity of the conclusions. Our categorization permitted us to manipulate content and context independently from the validity of the conclusions in five categories that could be independently manipulated. For example, we predicted different invalid conclusions for our intersection and disjunction conditions. The psychology of reasoning is supposed to predict what conclusion human beings will produce from a given set of premises, not only whether or not they will give the valid conclusion.

Another advantage is that the categorization of contents in terms of TCRR is useful in improving the strength of the mental models theory against criticism. We will examine two criticisms related to content effects in reasoning. Newstead and Evans (1993; see also Newstead et al., 1992) have recently claimed that the mental model theory cannot explain two pieces of evidence about belief bias. First, as we have mentioned, they remark on the lack of belief effect in multiple-model-determinate problems found in Newstead et al. (1992, Experiments 4 and 5) and Oakhill et al. (1989) as falsifying evidence against the model theory. However, it may be just a categorization problem arising from the use of believability as the content and context measure in syllogistic reasoning. Indeed, the "believable" version of the problems in Newstead et al. (1992, Experiment 4) used the valid conclusions Some pigeons are not fish or Some salmon are not birds. According to the TCRR categorization system, these represent disjunctions, so the representation of the concepts' relations in these statements (we will use the concepts of the former) would be

$\begin{array}{rr}\text { pigeon } & -f i s h \\ \neg \text { pigeon } & \text { fish } \\ - \text { pigeon } & \neg f i s h\end{array}$

- which is functionally equivalent to a universal negative conclusion and not to the existential negative used in the experiment. When this is taken into account, it is not surprising that this content produces no facilitation at all for this problem, but a reliable difference when the tested conclusion is a universal negative (Newstead et al., Experiment 1). In our Experiment 2, we provide an example on how to establish differences in a problem with an existential negative valid conclusion. In fact, Newstead et al.'s "believable" problems are similar to our disjunction condition, where we found a pattern of responses very similar to that in the abstract condition.

The second main criticism proposed by Newstead and Evans (1993) and applied to the mental model theory explanation of belief bias is the case of single-model problems. The measurable effects of beliefs in these problems makes it difficult to maintain the traditional mental-model explanation of this effect. Since there are no alternative models to consider, no effect can be predicted. Some ad hoc hypotheses have been introduced. Oakhill et al. (1989) postulated a "conclusion filtering" mechanism, and Newstead et al. (1992), a search for nonexistent alternative models. Both mechanisms are difficult to accommodate to existing data (Oakhill \& Garnham, 1993), and both provide a poor 
service to the general theory of mental models by approximating predictions for single-model problems to that of multiple-model problems. Indeed, if people search for alternative models in both single- and multiple-model problems, the difference between both kinds of problem will be small. However, the use of TCRR to study relations in both premises and conclusions shows clearly how different concept relations lead to different models, and moreover, how content-based differences are predicted for any kind of problem.

Another general criticism comes from the supporters of mental logic theory. Rips (1986, p. 279) argued that "content effects can't be used as an argument for the superiority of (these sorts of) mental models over inference schemata." This argument is based on the idea that representations in terms of mental models are as abstract as those of logical inference rules. Similarly, Smith, Langston, and Nisbett (1992, p. 29) stated that "there is no obvious reason why one cannot construct a mental model as readily for an unfamiliar item as for a familiar one, or as readily for an abstract item as a concrete one." However, a semantic mental model theory in which the meaning of the concepts included in the problem does not play a central role would be unthinkable. In this paper, we specify how the process of building mental models takes into account the conceptual relations expressed in the problem. Therefore, we have demonstrated that there is not the slightest evidence to justify the claim of abstractness in mental model theory. Moreover, we have demonstrated that from two problems with the same logical structure, content and context could lead to different predictable patterns of response, so that reasoning is not logically determined. There might be a reasoning theory in which, from a given logical structure, subjects completing all the reasoning stages should get the logically correct answer. Such a theory could explain the deviations from logical validity as errors. However, a semantic theory that is not based on formal rules of inference should explain responses of all kinds, as the result of different combinations in the use of the mechanisms of the theory. The aim of this paper has been to specify those mechanisms for problems including different types of concepts. Within a non-formal-rulebased system, the process does not necessarily lead to logical validity every time it is accurately finished. The categorization presented here makes it clear to us that the logically correct response for each type of problem is just one of the possible results produced by the mental model manipulation with some conceptual relationships. Therefore, correct conclusions occur when there is a functional equivalence between concept relationships and task demands. This is the cause of the logical appearance and rational nature of most human deductions.

\section{REFERENCES}

BADDELEY, A. D. (1986). Working memory. Oxford: Oxford University Press, Clarendon Press.

BARSTON, J. L. (1986). An investigation into belief biases in reasoning. Unpublished doctoral dissertation, University of Plymouth.
Braine, M. D. S. (1978). On the relation between the natural logic of reasoning and standard logic. Psychological Review, 85, 1-21.

Braine, M. D. S., \& Rumain, B. (1983). Logical reasoning. In P. H. Mussen (Ed.), Carmichael's Manual of child psychology (Vol. 3, pp. 263-340). New York: Wiley.

BYRNE, R. M. J. (1989). Suppressing valid inferences with conditionals. Cognition, 31, 61-83.

Cheng, P. W., \& Holyoak, K. J. (1985). Pragmatic reasoning schemas Cognitive Psychology, 17, 391-416.

Evans, J. Sт. B. T. (1989). Bias in human reasoning: Causes and consequences. Hove, U.K.: Erlbaum.

Evans, J. ST. B. T. (1993). The mental model theory of conditional reasoning: Critical appraisal and revision. Cognition, 48, 1-20.

Evans, J. St. B. T., Barston, J. L., \& Pollard, P. (1983). On the conflict between logic and belief in syllogistic reasoning. Memory \& Cognition, 11, 295-306.

Evans, J. St. B. T., Newstead, S. E., \& Byrne, R. M. J. (1993). Human reasoning: The psychology of deduction. Hillsdale, NJ: Erlbaum.

Garnham, A. (1981). Mental models as representations of text. Memory \& Cognition, 9, 560-565.

GlenberG, A. M., Meyer, M., \& Linden, K. (1987). Mental models contribute to foregrounding during text comprehension. Journal of Memory \& Language, 26, 69-83.

GrigGS, R. A., \& Cox, J. R. (1982). The elusive thematic-materials effects in Wason's selection task. British Journal of Psychology, 73, 407-420.

JoHNSON-LAIRD, P. N. (1983). Mental models: Towards a cognitive science on language, inference, and consciousness. Cambridge: Cambridge University Press.

Johnson-Laird, P. N. (1995). Inference and mental models. In S. E. Newstead \& J. St. B. T. Evans (Eds.), Perspectives on thinking and reasoning: Essays in honour of Peter Wason (pp. 115-146). Hove, U.K.: Erlbaum.

Johnson-Laird, P. N., \& Bara, B. G. (1984). Syllogistic inference. Cognition, 16, 1-62.

JohnSon-Laird, P. N., \& Byrne, R. M. J. (1991). Deduction. Hillsdale, NJ: Erlbaum.

Johnson-Laird, P. N., Byrne, R. M. J., \& Schaeken, W. (1992). Propositional reasoning by model. Psychological Review, 99, 418-439.

JoHnSON-LaiRd, P. N., \& STEEDMAN, M. J. (1978). The psychology of syllogisms. Cognitive Psychology, 10, 64-99.

LEGRENZI, P. (1970). Relations between language and reasoning about deductive rules. In G. B. Flores d'Arcais \& W. J. M. Levelt (Eds.), $A d$ vances in psycholinguistics (pp. 322-333). Amsterdam: Elsevier, North-Holland.

Manktelow, K. I., \& Evans, J. St. B. T. (1979). Facilitation of reasoning by realism: Effect or non-effect? British Journal of Psychology, 71, 227-231.

MARCOVITS, H. (1984). Awareness of the "possible" as a mediator of formal thinking in conditional reasoning problems. British Journal of Psychology, 75, 367-376.

MARCoviTs, H. (1985). Incorrect conditional reasoning among adults: Competence or performance? British Journal of Psychology, 76, 241-247.

Marcovits, H. (1988). Conditional reasoning, representation, and empirical evidence on a concrete task. Quarterly Journal of Experimental Psychology, 40A, 483-495.

Marcus, S. L., \& RIPS, L. J. (1979). Conditional reasoning. Journal of Verbal Learning \& Verbal Behavior, 18, 199-223.

Newstead, S. E., \& Evans, J. ST. B. T. (1993). Mental models as an explanation of belief bias effects in syllogistic reasoning. Cognition, 46, 93-97.

Newstead, S. E., Pollard, P., Evans, J. St. B. T., \& Allen, J. L. (1992). The source of belief bias effects in syllogistic reasoning. Cognition, 45, 257-284.

OAKHILl, J. V., \& GarNhaM, A. (1993). On theories of belief bias in syllogistic reasoning. Cognition, 46, 87-92.

Oakhill, J.V., Garnham, A., \& Johnson-Laird, P. N. (1990). Belief bias effects in syllogistic reasoning. In K. J. Gilhooly, M. T. G. Keane, R. H. Logie, \& G. Erdos (Eds.), Lines of thinking: Reflections on the psychology of thought. Vol. 1: Representation, reasoning, analogy and decision making (pp. 125-138). Chichester, U.K.: Wiley. 
OAKHILL, J. V., \& JOHNSON-LAIRD, P. N. (1985). The effects of belief on the spontaneous production of syllogistic conclusions. Quarterly Journal of Experimental Psychology, 37A, 553-570.

Oakhill, J. V., Johnson-Laird, P. N., \& Garnham, A. (1989). Believability and syllogistic reasoning. Cognition, 31, 117-140.

OSHERSON, D. (1975). Logic and models of logical thinking. In R. J. Falmagne (Ed.), Reasoning: Representation and process (pp. 81-91). New York: Wiley.

RiPs, L. J. (1986). Mental muddles. In M. Brand \& R. M. Harnish (Eds.) Problems in the representation of knowledge and belief (pp. 258-286). Tucson: University of Arizona Press

RIPS, L. J., \& MARCUS, S. L. (1977). Suppositions and the analysis of conditional sentences. In M. A. Just \& P. A. Carpenter (Eds.), Cognitive processes in comprehension (pp. 185-220). New York: Wiley.
Rumain, B., Connell, J., \& Braine, M. D. S. (1983). Conversational comprehension processes are responsible for reasoning fallacies in children as well as adults. Developmental Psychology, 19, 471-481.

Santamaría, C. (1989). Modelos mentales y razonamiento semántico: el silogismo. Cognitiva, 2(2), 21-36.

SCHEFFE, H. A. (1960). The analysis of variance. New York: Wiley. Smith, E. E., Langston, C., \& Nisbett, R. E. (1992). The case for rules in reasoning. Cognitive Science, 16, 1-40.

WASON, P. C. (1966). Reasoning. In B. Foss (Ed.), New horizons in psychology (pp. 135-151). Harmondsworth, U.K.: Penguin.

(Manuscript received February 21, 1995; revision accepted for publication June 16,1995 .) 